\title{
P16 Expression Independent of Human Papilloma Virus in Egyptian Patients with Head and Neck Squamous Cell Carcinoma: A Retrospective Study
}

\author{
Dina RD. Ibrahim ${ }^{1}$, Reham M. Faheim ${ }^{2}$, Mohamed R. Kelany ${ }^{3}$, Nahla M. Awad ${ }^{4}$, Hala M. El-Sallaly ${ }^{5}$, \\ Nashwa N. El-Khazragy ${ }^{6}$ \\ ${ }^{1,2,3}$ Clinical Oncology Department, Ain-Shams University \\ ${ }^{4,5}$ Histopathology Department, Ain-Shams University \\ ${ }^{6}$ Clinical Pathology department, Ain-Shams University
}

\begin{abstract}
Background: the prognostic role of human papillomavirus (HPV) associated p16 expression in oropharyngeal squamous cell carcinoma (OPSCC) is well established; however data are less on the prognostic significance of p16 expression independent of HPV status in non-oropharyngeal SCC (non-OPSCC). We evaluated the expression of p16 in different sites of head and neck squamous cell carcinoma (HNSCC) in Egyptian patients in correlation to the relevant clinical characteristics and treatment outcome. Methods: Fortytwo paraffin blocks of HNSCC were collected and immunostained for p16. Clinical data were extracted from the charts from 2011-2016, at the Clinical Oncology Department- Ain-Shams University, and analyzed for the p16 status. P16 positive case was diagnosed when there was positive diffuse nuclear and cytoplasmic staining of the majority (70\%) of tumor cells. Results: Twenty-five (59.5\%) out of 42 patients were positive for $p$ 16, while 17 were negative (40.5\%). P16 + and p16 - patients had no differences in age, smoking, tumor site, tumor grade, and stage. Expression of p16 was higher in patients with Eastern Cooperative Oncology Group (ECOG) performance 1 and 2 (p 0.045). No correlation was observed between p16 status and type of treatment or recurrence. P16+ patients tended to have higher incidence of metastases (p 0.062). The p16 status did not affect the disease free survival (DFS) or the overall survival (ORS). Conclusion: Most of our patients had positive p16 expression. We could not show significant correlation between p16 status and clinical characteristics, and outcome. Large studies are needed to further investigate the prognostic role of p16 as a marker of HPV infection in non-OPSCC.
\end{abstract}

Keywords: Head and neck squamous cell carcinoma, human papillomavirus, immunohistochemistry, p16.

\section{Introduction}

The role of high-risk human papilloma viruses (HPVs) in the development of head and neck squamous cell carcinomas (HNSCCs) is well known, where around $30 \%$ of patients are positive for the high-risk HPVs [1-3]. Multiple studies have reported that the high risk subtypes HPV 16 and 18 have major role in the etiology and better prognosis of overall survival and locoregional control in oropharyngeal squamous cell carcinoma (OPSCC) [4-6]. The high-risk subtype HPV 16 represents more than $85 \%$ of HPV positive tumors in HNSCC [7].

Clinically p 16 immunohistochemistry IHC is accepted as a surrogate biomarker for the presence of HPV in OPSCC based on the high concordance between this method and other HPV detection methods such as type specific HPVDNA detection by situ hybridization (ISH) [8], [9]. However, in non-OPSCCs which have lower incidence of HPV association, p 16 is not as useful as an HPV surrogate maker. This in part could be due to tumor-site differences and also the use of diverse detection testing [10], [11]. P16 is present in normal cells at a low level. P16 IHC assesses the protein product of the tumor suppressor gene CDKN2A which is lost in the majority of HPV positive tumors and is expressed in HPV negative tumors [12]. P16 has a key role in cell cycle control where it represses the $\mathrm{D}$ cyclins by phosphorylation of the retinoblastoma tumor suppressor protein (RB1). In HPV infected cancer cells, E7 viral oncoproteins degrade RB1 and enhance p16 expression [13], although RB1 loss via mutations can also occur in HPV negative HNSCCs leading to p16 expression in $5-8 \%$ of these HPV negative tumors [14]. Thus, p16 expression is not specific for HPV associated tumors, for example the OSCCs where the probability of p16 expression is low, the truepositive rate of p16 drops to $41.3 \%$ rendering p16 IHC an ineffective HPV surrogate diagnostic [15],[16]. P16 IHC has major advantages of being rapid, readily available and inexpensive technique [17], [18]. However, p16 IHC is not accepted as the gold standard due to its low specificity, where positive p16 is detected in 10-20\% of HPV negative tumors according to several studies [17], [19], [20].

In OPSCC, p16 overexpression is associated with improved ORS and local control, thus used as a prognostic marker [21]. Moreover, p16 expression has been suggested as an independent predictor of response to radiotherapy regardless of the HPV status in the oropharynx [22]. However, there is limited information regarding the incidence of p16 expression and its prognostic role in non-oropharyngeal HNSCC [23].Wilson et al [4] reported on patients with hypophayrngeal SCC with p16 expression who had poor ORS, locoregional control and shorter DFS. They showed that hypophayrngeal SCC p16 positive patients are generally HPV negative which suggests a different mechanism for $\mathrm{p} 16$ expression. Satgunaseelan et al [24] evaluated the role and incidence of p16 in 215 cases of oral squamous cell carcinoma (OSCC) using p1 16 IHC and HPV ISH. Thirtyseven $(17.2 \%)$ cases showed p16 expression without association with HPV. P16 expression was seen in early 


\section{International Journal of Science and Research (IJSR) \\ ISSN (Online): 2319-7064}

Index Copernicus Value (2015): 78.96 | Impact Factor (2015): 6.391

stage OSCCs and was associated with better survival following surgery and radiotherapy.

We aimed to study the prevalence of p16 expression in different sites HNSCC, and its prognostic impact on clinicopathologic factors and outcome.

\section{Material and Methods}

A total of paraffin blocks of 42 patients who were treatment naive, presented to the Clinical Oncology department, AinShams University, were included in this study. We studied patients from 2011 to 2016 to ensure a homogenous treatment protocol. The approval of the Research Ethical Committee at Faculty of Medicine, Ain-Shams University was obtained. All the patients had a histologic diagnosis of SCC of all sites. All the patients had conventional SCC except for two basaloid and two undifferentiated SCC variants. The patients were staged according to the American Joint Committee on Cancer (AJCC) $7^{\text {th }}$ edition 2010. The paraffin blocks were examined for presence or absence of p16 by immunohistochemistry. Then data were analyzed in relation to the clinical parameters including age, sex, smoking history, tumor site, grade, stage, lymph node metastasis, treatment, ORS, and DFS.

\section{Immunohistochemical (IHC) study}

Archived formalin-fixed paraffin-embedded tumor blocks were retrieved and sectioned at $3 \mathrm{~mm}$ thickness, and mounted on microscopic positive charged slides. Antigen retrieval for all tissue sections in $10 \mathrm{ml}$ citrate buffer, $\mathrm{pH}$ 6.0, for 10-20 min was done. After thermal treatment, jars with buffer and slides were allowed to cool for $20 \mathrm{~min}$ at room temperature. Sections were rinsed gently with buffer or deionized water. The tissue sections were not allowed to dry out during the treatment or during the following immunohistochemical staining procedure. Serial sections were immunostained for CD16 using CD16 (DJ130c): sc20052 Mouse Monoclonal Antibodies manufactured by Santa Cruz Biotechnology Primary antibodies for CD16+ were incubated $120 \mathrm{~min}$, at room temperature. The reaction developed was detected by the brown stain of 2.3 DAB containing $0.01 \% \mathrm{H} 2 \mathrm{O} 2$. The sections were counterstained with hematoxylin, then dehydrated, and mounted with distyrene, plasticizer, xylene (DPX) standard resin (Lamb Ltd.; London, UK), and finally examined by a bright-field light microscope. Appropriate positive controls were used (tonsils for CD16+) to judge the effectiveness of the staining technique, and mouse immunoglobulin-G (Ig-G) antibodies were used as negative controls. The slides were studied at Ain Shams University Hospital Early cancer detection Unit by the two pathologists who were blinded to the cases and controls.

\section{Interpretation of IHC results}

Semi-quantitative assessment was used to estimate the percentage of tumor cells that stained, with p16 positive case diagnosed when there was positive diffuse nuclear and cytoplasmic staining of the majority $(70 \%)$ of tumor cells, regardless of intensity of staining i.e. cell was assumed to be positively stained if it showed any level of staining above background levels with different patterns nuclear, membranous or cytoplasmic (Figure1). Negative case was diagnosed when there was complete absence of staining in all tumor cells or membranous/cytoplasmic staining of rare, isolated tumor cells (up to $40 \%$ stained tumor cells) [25].

\section{Statistics}

Data were analyzed using the Statistical Package for Social Science (IBM SPSS) version 20. The qualitative data were presented as number and percentages while quantitative data were presented as mean, standard deviations and ranges when their distribution found parametric while nonparametric data were presented as median with interquartile range (IQR). The comparison between two groups with qualitative data was done using Chi-square test. The comparison between two independent groups with quantitative data and non-parametric distribution was done using Mann-Whitney test. DFS was calculated from the date of surgery or (first treatment received) to date of first disease recurrence, or to the date of death or last follow up if there was no disease recurrence. ORS was calculated from the date of surgery or (first treatment received) to date of death or last follow up. The confidence interval was set to $95 \%$ and the margin of error accepted was set to $5 \%$. So, the pvalue was considered significant as the following: $\mathrm{p}>0.05$ : Non significant, $\mathrm{p}<0.05$ : Significant, and $\mathrm{p}<0.01$ : Highly significant.

\section{Results}

\section{Patient's characteristics}

The demographic and clinical criteria of the 42 patients diagnosed with HNSCC are summarized in (Table 1).

\section{Results in relation to $\mathbf{p} 16$ expression}

Nuclear and cytoplasmic expression of p16 was observed in $25(59.5 \%)$ cases. Correlation analysis revealed nonsignificant link between p16 expression and patient's demographics except for the ECOG status (p 0.045). Positive p16 was more frequent in male gender (M: F 4:1), less frequent in the age range 45-65 years. High incidence of p16 expression was observed in smokers. (Table 2)

Laryngeal SCC (LSCC) was the most frequent tumor site in p16 positive tumors in contrast to nearly equal rates seen in other subsites (oral cavity and pharynx) (p 0.840). P16 positive tumors were more likely to be of grade II, T3, and stage III/IV as compared to p16 negative tumors, however; these differences were not significant ( $p \geq 0.05$ ) (Table 2).

We could not demonstrate a significant correlation between p16 expression and different forms of treatment or response to treatment ( $p \geq 0.05$ ), as illustrated in (Table2). Definitive combined chemo-radiotherapy was the commonest treatment $16 / 42(38.1 \%)$ followed by combined surgical resection and chemo-radiotherapy $11 / 42(26.2 \%)$.

The patients were followed up for 41 months duration. 25/42 patients $(59.5 \%, \mathrm{p} 0.390)$ achieved CR and PR was obtained in $6 / 42(14.3 \%, \mathrm{p} 0.390) .5 / 42$ patients $(11.9 \%)$ developed

\section{Volume 6 Issue 7, July 2017




\section{International Journal of Science and Research (IJSR) \\ ISSN (Online): 2319-7064 \\ Index Copernicus Value (2015): 78.96 | Impact Factor (2015): 6.391}

local recurrence while $12 / 42$ patients died; the mortality rate was $28.6 \%$. The mean duration of ORS and DFS were 11.9, 9.98 months respectively in all patients (Table 1). The mean ORS of p16+ve and p16-ve patients was 13.84 and 10.27 with a statistical difference (p 0.022). The mean DFS of p16 negative and positive patients was 12.14 months and 8.52 months, respectively (p 0.009, Table 2).

The 2-years follow up probability of ORS and DFS didn't differ for p16 negative or positive tumors; Kaplan-Meier survival analysis showed non-significant mean ORS of p16 positive tumors of 12.34 versus 18.63 in p16 negative

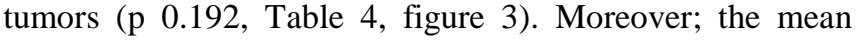
DFS of p16 positive and negative tumors was 19.42 and
27.32 months respectively with no statistically significant difference ( $p \geq 0.05$, Figure 3 ).

Relationship between ORS, DFS and different treatment regimens in p16 negative and positive $H N$ tumors (Table5, figure 1)

Chi-square test showed no statistical difference between OS in p16 positive HNSCC and different treatment regimens used ( $p$ $\geq 0.05$, Table 3). Kaplan Meier survival analysis of ORS at the end of first-line treatment and type of regimens administered in p16 negative and p16 positive HN tumors showed that the type of therapy has no impact on ORS, DFS between p16 negative or positive tumors (Table 5).

Table 1: Patients Demographic/Clinico-Pathological Features

\begin{tabular}{|c|c|c|c|}
\hline Variable & 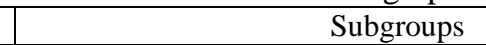 & Parameter & Frequencies \\
\hline Age (years) & & $\begin{array}{l}\text { Mean } \pm \text { SD } \\
\text { Range }\end{array}$ & $\begin{array}{c}58.93 \pm 11.938 \\
26.0-85.0\end{array}$ \\
\hline Age subgroups (years) & $\begin{array}{l}<45 \\
46-65 \\
>65\end{array}$ & $\mathrm{n}(\%)$ & $\begin{array}{l}6(14.3 \%) \\
21(50 \%) \\
15(35.7)\end{array}$ \\
\hline Gender $(\mathrm{M} / \mathrm{F})$ & $\begin{array}{l}\text { Males } \\
\text { Females }\end{array}$ & & $\begin{array}{c}36(85.7 \%) \\
6(14.3 \%)\end{array}$ \\
\hline Smoking & $\begin{array}{l}\text { Non smoker } \\
\text { Smoker }\end{array}$ & & $\begin{array}{l}13(31 \%) \\
29(69 \%)\end{array}$ \\
\hline Tumor Subsite & $\begin{array}{l}\text { Oral cavity, Maxilla, Paranasal sinus } \\
\text { Pharynx, Oropharynx } \\
\text { Larynx }\end{array}$ & & $\begin{array}{c}8(19 \%) \\
9(21.4 \%) \\
25(59.5 \%) \\
\end{array}$ \\
\hline Tumor grade & $\begin{array}{l}\text { Grade I } \\
\text { Grade II } \\
\text { Grade III } \\
\end{array}$ & & $\begin{array}{c}2(4.8 \%) \\
26(61.9 \%) \\
14(33.3 \%)\end{array}$ \\
\hline Tumor Stage & \begin{tabular}{|l|} 
Stage I \\
Stage II \\
Stage III \\
Stage IV
\end{tabular} & & $\begin{array}{c}2(4.8 \%) \\
5(11.9 \%) \\
19(45.2 \%) \\
16(38.1 \%)\end{array}$ \\
\hline Tumor size $\mathrm{T}$ & \begin{tabular}{l|} 
T1 \\
T2 \\
T3 \\
T4 \\
\end{tabular} & & $\begin{array}{c}2(4.8 \%) \\
12(28.6 \%) \\
24(57.1 \%) \\
4(9.5 \%) \\
\end{array}$ \\
\hline Lymph nodes $\mathrm{N}$ & $\begin{array}{l}\text { N0 } \\
\text { N1 } \\
\text { N2 } \\
\text { N3 }\end{array}$ & & $\begin{array}{c}19(45.2 \%) \\
11(26.2 \%) \\
10(23.8 \%) \\
2(4.8 \%) \\
\end{array}$ \\
\hline Metastasis M & $\begin{array}{l}\text { No } \\
\text { Yes }\end{array}$ & & $\begin{array}{c}39(92.1 \%) \\
3(7.1 \%)\end{array}$ \\
\hline Form of Treatment & $\begin{array}{l}\text { Surgery only } \\
\text { Chemotherapy } \\
\text { Radiotherapy } \\
\text { Combined chemo-radiotherapy } \\
\text { Surgery + chemotherapy } \\
\text { Surgery + radiotherapy } \\
\text { Surgery + chemo-radiotherapy }\end{array}$ & & $\begin{array}{c}1(2.4 \%) \\
3(7.1 \%) \\
6(14.3 \%) \\
16(38.1 \%) \\
1(2.4 \%) \\
4(9.5 \%) \\
11(26.2 \%) \\
\end{array}$ \\
\hline Response to treatment & $\begin{array}{l}\text { Complete remission }(\mathrm{CR}) \\
\text { Partial remission }(\mathrm{PR}) \\
\text { Stable disease }(\mathrm{SD}) \\
\text { Progressive disease }(\mathrm{PD})\end{array}$ & & $\begin{array}{l}25(59.5 \%) \\
6(14.3 \%) \\
5(11.9 \%) \\
6(14.3 \%)\end{array}$ \\
\hline Local recurrence & $\begin{array}{l}\text { No } \\
\text { Yes }\end{array}$ & & $\begin{array}{c}37(88.1 \%) \\
5(11.9 \%)\end{array}$ \\
\hline DFS (months) & & $\begin{array}{l}\text { Mean } \pm \text { SD } \\
\text { Range }\end{array}$ & $\begin{array}{c}9.98 \pm 8.93 \\
0-41.0\end{array}$ \\
\hline ORS (months) & & $\begin{array}{l}\text { Mean } \pm \text { SD } \\
\text { Range }\end{array}$ & $\begin{array}{c}11.9 \pm 9.58 \\
1-41.0\end{array}$ \\
\hline Patient status after 2 years & $\begin{array}{l}\text { Alive } \\
\text { dead }\end{array}$ & & $\begin{array}{l}30(71.4 \%) \\
12(28.6 \%)\end{array}$ \\
\hline
\end{tabular}

Volume 6 Issue 7, July 2017 www.ijsr.net 


\section{International Journal of Science and Research (IJSR) \\ ISSN (Online): 2319-7064}

Index Copernicus Value (2015): 78.96 | Impact Factor (2015): 6.391

Table 2: Association between p16 status and Clinico-pathological Features

\begin{tabular}{|c|c|c|c|c|c|}
\hline Variable & Subgroups & $\begin{array}{c}\text { P16 negative } \\
\mathrm{n}=17 \%(40.5 \%)\end{array}$ & $\begin{array}{c}\text { P16 positive } \\
\mathrm{n}=25 \%(59.5 \%)\end{array}$ & $\begin{array}{l}\text { Person's } \\
\text { chi-square }\end{array}$ & $P$ value \\
\hline \multicolumn{6}{|l|}{ Patient Characteristics } \\
\hline Gender & \begin{tabular}{|l|} 
Males \\
Females
\end{tabular} & $\begin{array}{c}16 \\
1\end{array}$ & $\begin{array}{c}20 \\
5\end{array}$ & 1.647 & 0.206 \\
\hline Age (years) & $\begin{array}{l}\leq 45 \\
46-65 \\
>65\end{array}$ & $\begin{array}{l}3 \\
7 \\
7\end{array}$ & $\begin{array}{c}3 \\
14 \\
8\end{array}$ & 0.909 & 0.635 \\
\hline Smoking & \begin{tabular}{|l|} 
Non-smoker \\
Smoker
\end{tabular} & $\begin{array}{c}5 \\
12 \\
\end{array}$ & $\begin{array}{c}8 \\
17 \\
\end{array}$ & 0.032 & 0.517 \\
\hline ECOG & $\begin{array}{l}0 \\
1 \\
2 \\
\end{array}$ & $\begin{array}{c}2 \\
15 \\
0\end{array}$ & $\begin{array}{c}2 \\
18 \\
5\end{array}$ & 8.041 & 0.045 \\
\hline \multicolumn{6}{|l|}{ Pathological Characteristics } \\
\hline Tumor subsite & $\begin{array}{l}\text { Oral cavity, Maxilla, Paranasal sinus } \\
\text { Pharynx, Oropharynx } \\
\text { Larynx }\end{array}$ & $\begin{array}{c}3 \\
3 \\
11\end{array}$ & $\begin{array}{c}5 \\
6 \\
14\end{array}$ & 0.349 & 0.840 \\
\hline Tumor 1 grade & $\begin{array}{l}\text { I } \\
\text { II } \\
\text { III }\end{array}$ & $\begin{array}{c}1 \\
10 \\
6\end{array}$ & $\begin{array}{c}1 \\
16 \\
8\end{array}$ & 0.152 & 0.927 \\
\hline Tumor stage & $\begin{array}{l}\text { Stage I } \\
\text { Stage II } \\
\text { Stage III } \\
\text { Stage IV }\end{array}$ & $\begin{array}{l}1 \\
3 \\
8 \\
5\end{array}$ & $\begin{array}{c}1 \\
2 \\
11 \\
11\end{array}$ & 1.45 & 0.693 \\
\hline Tumor size $\mathrm{T}$ & $\begin{array}{l}\text { T1 } \\
\text { T2 } \\
\text { T3 } \\
\text { T4 }\end{array}$ & $\begin{array}{l}1 \\
2 \\
3 \\
4\end{array}$ & $\begin{array}{c}1 \\
6 \\
16 \\
2\end{array}$ & 1.186 & 0.756 \\
\hline Lymph nodes $\mathrm{N}$ & $\begin{array}{l}\text { N0 } \\
\text { N1 } \\
\text { N2 } \\
\text { N3 }\end{array}$ & $\begin{array}{l}10 \\
3 \\
4 \\
0\end{array}$ & $\begin{array}{l}9 \\
8 \\
6 \\
2\end{array}$ & 3.322 & 0.345 \\
\hline Metastasis M & \begin{tabular}{|l|} 
No \\
Yes \\
\end{tabular} & $\begin{array}{c}17 \\
0\end{array}$ & $\begin{array}{c}22 \\
3\end{array}$ & 2.197 & 0.200 \\
\hline \multicolumn{6}{|l|}{ Treatment \& Follow up } \\
\hline Treatment Form & $\begin{array}{l}\text { Surgery only } \\
\text { Chemotherapy } \\
\text { Radiotherapy } \\
\text { Combined chemo-radiotherapy } \\
\text { Surgery + chemotherapy } \\
\text { Surgery + radiotherapy } \\
\text { Surgery + chemo-radiotherapy }\end{array}$ & $\begin{array}{l}1 \\
1 \\
2 \\
7 \\
0 \\
1 \\
5\end{array}$ & $\begin{array}{l}0 \\
2 \\
4 \\
9 \\
1 \\
3 \\
6\end{array}$ & 2.923 & 0.818 \\
\hline Response to Therapy & $\begin{array}{l}\text { CR } \\
\text { PR } \\
\text { SD } \\
\text { PD } \\
\end{array}$ & $\begin{array}{l}9 \\
4 \\
1 \\
3\end{array}$ & $\begin{array}{l}16 \\
2 \\
4 \\
3 \\
\end{array}$ & 3.012 & 0.390 \\
\hline Local Recurrence & \begin{tabular}{|l|} 
No \\
Yes \\
\end{tabular} & $\begin{array}{c}14 \\
3\end{array}$ & $\begin{array}{c}23 \\
2\end{array}$ & 0.898 & 0.317 \\
\hline Distant metastases & \begin{tabular}{|l|} 
No \\
Yes \\
\end{tabular} & $\begin{array}{c}17 \\
0\end{array}$ & $\begin{array}{c}20 \\
5\end{array}$ & 3.859 & 0.050 \\
\hline Patient status at 2 years FU & $\begin{array}{l}\text { Alive } \\
\text { Dead }\end{array}$ & $\begin{array}{c}12 \\
5\end{array}$ & $\begin{array}{c}18 \\
7\end{array}$ & 0.10 & 0.594 \\
\hline ORS months & Mean \pm SD & $\begin{array}{c}\text { Mean } \pm \text { SD } \\
13.84 \pm 12.68 \\
\end{array}$ & $\begin{array}{c}\text { Mean } \pm \text { SD } \\
10.27 \pm 6.82 \\
\end{array}$ & $\mathrm{~F}=5.69$ & 0.022 \\
\hline DFS months & Mean \pm SD & $\begin{array}{c}\text { Mean } \pm \text { SD } \\
12.14 \pm 12.51\end{array}$ & $\begin{array}{l}\text { Mean } \pm \text { SD } \\
8.52 \pm 5.04\end{array}$ & $\mathrm{~F}=7.52$ & 0.009 \\
\hline
\end{tabular}

Table 3: Association between type of treatment and survival in p16 positive patients

\begin{tabular}{|l|c|c|c|}
\hline \multicolumn{3}{|c|}{ Mean $^{\mathrm{a}}$ for survival Time } \\
\hline \multirow{2}{*}{ Treatment form } & \multicolumn{2}{|c|}{ 95\% Confidence Interval } & \multicolumn{2}{c|}{ Log Rank (Mantel-Cox) } \\
\cline { 2 - 4 } & Lower bound & Upper bound & \multicolumn{2}{c|}{ Chi-Square } & \\
\hline Chemotherapy only & 0.000 & 14.800 & \\
Radiotherapy only & 4.043 & 10.707 & \multirow{2}{*}{0.348} \\
Definitive Radio-chemotherapy & 9.669 & 21.759 & \\
Surgery followed by chemotherapy & 13.000 & 13.000 & \\
Surgery followed by radiotherapy & 2.838 & 21.095 & \\
Overall & 7.648 & 12.462 & \\
\hline
\end{tabular}

Volume 6 Issue 7, July 2017 www.ijsr.net

Licensed Under Creative Commons Attribution CC BY 


\section{International Journal of Science and Research (IJSR) \\ ISSN (Online): 2319-7064 \\ Index Copernicus Value (2015): 78.96 | Impact Factor (2015): 6.391}

a. Estimation is limited to the largest survival time.

Table 4: Cox survival analysis of effect of p16 status on OS and DFS

\begin{tabular}{|c|c|c|c|c|c|c|c|}
\hline \multicolumn{8}{|c|}{ Mean $^{\mathrm{a}}$ for survival Time } \\
\hline \multirow{2}{*}{ Factor } & \multirow{2}{*}{$\begin{array}{c}\text { P16 expression } \\
\text { subgroups }\end{array}$} & \multirow{2}{*}{ Estimate } & \multirow{2}{*}{ SE } & \multicolumn{2}{|c|}{ 95\% Confidence Interval } & \multicolumn{2}{|c|}{ Log Rank (Mantel-Cox) } \\
\hline & & & & Upper bound & Lower bound & Chi-Square & P-value \\
\hline \multirow{3}{*}{ Overall survival } & P16 negative & 18.631 & 4.087 & 10.620 & 26.643 & \multirow{3}{*}{1.704} & \multirow{3}{*}{0.192} \\
\hline & P16 positive & 12.849 & 1.839 & 9.245 & 16.453 & & \\
\hline & Overall & 15.424 & 2.092 & 11.323 & 19.525 & & \\
\hline \multirow{3}{*}{ Disease free survival } & P16 negative & 27.392 & 4.706 & 18.168 & 36.617 & \multirow{3}{*}{0.142} & \multirow{3}{*}{0.707} \\
\hline & P16 positive & 19.421 & 2.726 & 14.077 & 24.764 & & \\
\hline & Overall & 26.140 & 3.278 & 19.715 & 32.564 & & \\
\hline
\end{tabular}

a. Estimation is limited to the largest survival time if it is censored, $\mathrm{SE}=$ standard error, Estimate $=$ mean survival time.

Table 5: Kaplan Meier survival analysis of effect of treatment regimen on overall survival in p16 negative and p16 positive patients

\begin{tabular}{|c|c|c|c|c|c|c|c|c|c|c|c|}
\hline \multirow[t]{2}{*}{ Variable } & \multirow[t]{2}{*}{ Group } & \multicolumn{3}{|c|}{ P16 positive $\mathrm{HNC}$} & \multicolumn{2}{|c|}{$\begin{array}{c}\text { Log Rank (Mantel- } \\
\text { Cox) }\end{array}$} & \multicolumn{3}{|c|}{ P16 negative HNC } & \multicolumn{2}{|c|}{$\begin{array}{c}\text { Log Rank (Mantel- } \\
\text { Cox) }\end{array}$} \\
\hline & & Total & Event & Censored & Chi-Square & $\mathrm{P}$ value & Total & Event & Censored & Chi-Square & $\mathrm{P}$ value \\
\hline \multirow{3}{*}{$\begin{array}{l}\text { Definitive Chemo-radio } \\
\text { therapy }\end{array}$} & Yes & 5 & 1 & 4 & \multirow{3}{*}{0.075} & \multirow{3}{*}{0.784} & 2 & 0 & 2 & \multirow{3}{*}{1.021} & \multirow{3}{*}{0.312} \\
\hline & No & 20 & 6 & 14 & & & 15 & 5 & 10 & & \\
\hline & All & 25 & 7 & 18 & & & 17 & 5 & 12 & & \\
\hline \multirow{3}{*}{ Chemotherapy alone } & Yes & 2 & 0 & 2 & \multirow{3}{*}{0.439} & \multirow{3}{*}{0.508} & 4 & 1 & 3 & \multirow{3}{*}{0.010} & \multirow{3}{*}{0.921} \\
\hline & No & 23 & 7 & 16 & & & 13 & 4 & 9 & & \\
\hline & All & 25 & 7 & 18 & & & 15 & 5 & 12 & & \\
\hline \multirow{3}{*}{ Radiotherapy alone } & Yes & 5 & 1 & 4 & \multirow{3}{*}{0.197} & \multirow{3}{*}{0.697} & 2 & 0 & 2 & \multirow{3}{*}{0.821} & \multirow{3}{*}{0.325} \\
\hline & No & 20 & 6 & 14 & & & 15 & 5 & 10 & & \\
\hline & All & 25 & 7 & 18 & & & 0 & 5 & 12 & & \\
\hline \multirow{3}{*}{ Surgery } & Yes & 0 & 0 & 0 & \multirow{3}{*}{ A } & \multirow{3}{*}{ NA } & 2 & 0 & 2 & \multirow{3}{*}{0.401} & \multirow{3}{*}{0.526} \\
\hline & No & 25 & 7 & 18 & & & 15 & 5 & 10 & & \\
\hline & All & 25 & 7 & 18 & & & 17 & 5 & 12 & & \\
\hline \multirow{3}{*}{$\begin{array}{l}\text { Surgery followed by } \\
\text { Chemotherapy }\end{array}$} & Yes & 1 & 0 & 1 & \multirow{3}{*}{0.776} & \multirow{3}{*}{0.378} & 0 & 0 & 0 & \multirow{3}{*}{ NA } & \multirow{3}{*}{ NA } \\
\hline & No & 24 & 7 & 17 & & & 17 & 5 & 12 & & \\
\hline & All & 25 & 7 & 18 & & & 17 & 5 & 12 & & \\
\hline \multirow{3}{*}{$\begin{array}{l}\text { Surgery followed by } \\
\text { Radiotherapy }\end{array}$} & Yes & 3 & 1 & 2 & \multirow{3}{*}{0.847} & & 1 & 0 & 1 & & \\
\hline & $\mathrm{No}$ & 22 & 6 & 16 & & 0.327 & 16 & 5 & 11 & 0.129 & 0.719 \\
\hline & All & 25 & 7 & 18 & & & 17 & 5 & 12 & & \\
\hline
\end{tabular}

Event: dead outcome, censored: alive outcome, NA: No comparison analysis is performed because one of the groups has no patients.

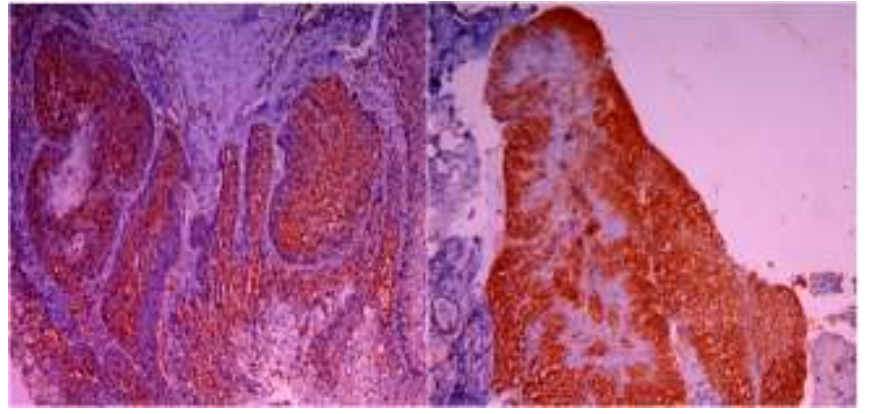

Figure 1: Strong, diffuse nuclear and cytoplasmic p16 immunostaining

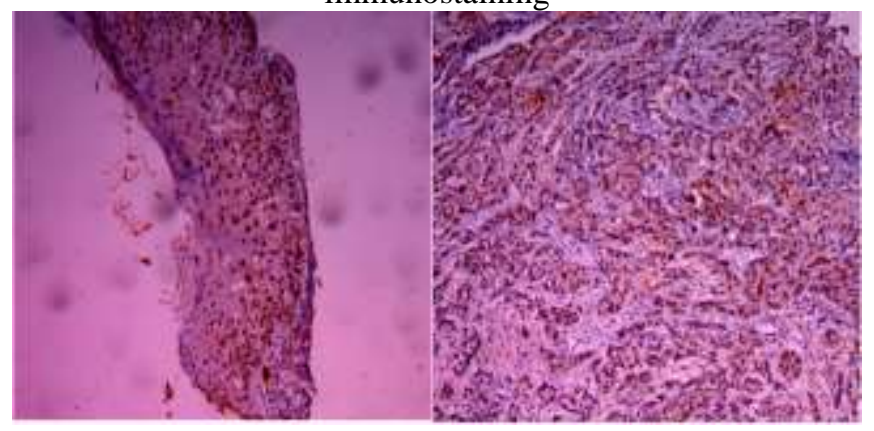

Figure 2: Strong, diffuse nuclear p16 immunostaining

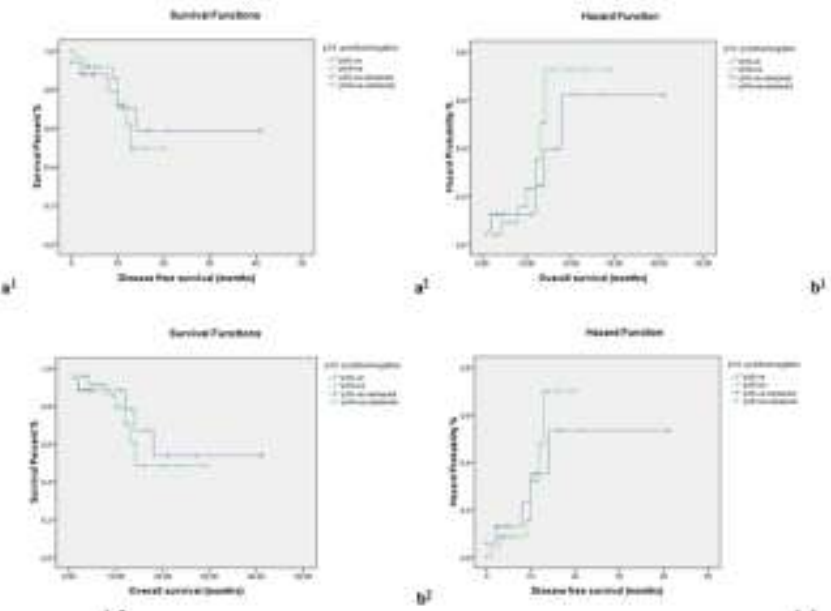

Figure 3: $[(a 1,2)$ Kaplan-Meier plots showing overall survival (OS) and hazards probability (HP), (b1, 2) KaplanMeier plots showing disease free survival (DFS) and hazards probability (HP)] in p16 positive and negative HNSCC

\section{Volume 6 Issue 7, July 2017 www.ijsr.net}




\section{International Journal of Science and Research (IJSR) \\ ISSN (Online): 2319-7064 \\ Index Copernicus Value (2015): 78.96 | Impact Factor (2015): 6.391}

\section{Discussion}

In the present study we assessed the prevalence of p16 in different subsites of HNSCC patients and the correlation between p16 expression, clinico-pathologic and treatment characteristics. Our patients sample consisted of SCC mainly of the larynx, hypopharynx, and oral cavity. We detected a higher incidence of positive p16 59.5\% versus p16 negative expression in $40.5 \%$ of the patients. According to literature reviews the HPV prevalence in LSCC is about $24 \%$, based on PCR detection methods [10], [11], and 13-29\% in HSCC [2], [26]. Similarly, Meshman et al [23] studied 31 patients with SCC of the larynx (23) and hypopharynx (8), where $58 \%$ of patients were p16- negative; and $45.2 \%$ were $\mathrm{p} 16$ positive. In a similar cohort with multiple sites HNSCC, positive p16 was observed in 59/75 (78.67\%), while 11 (21.33\%) cases were p16 negative [27]. Lassen et al [28] studied 1249 patients diagnosed with advanced SCC of the oropharynx (OPSCC), larynx and hypopharynx (nonOPSCC). They demonstrated higher frequency of p16 + in OPSCC (425/815) than in non-OPSCC (65/479), $\mathrm{p}<.0001)$. Positive p16 was detected in $14 \%$ of both larynx and hypopharynx carcinomas.

While the favorable prognostic significance of HPV +/p 16 + expression is well established in OPSCC, the prognostic significance of HPV and/or p 16 expression in non-OPSCC is not clearly delineated [21], [20], [29]. Our study cohort consisted mainly of SCC of the larynx, hypopharynx, and oral cavity $(57.1 \%, 16.7 \%$, and $14.3 \%$ respectively) while only $4.8 \%$ (2/42 patients) had OPSCC. Although the p16+ and p16 - patients were comparable in the various clinical and treatment parameters: age, gender, primary tumor site, T-stage, $\mathrm{N}$ - stage, and treatment modality, we found no statistical significance between p16 expression and these various factors except for the performance status (p 0.045). Different anatomic sites of HNSCC have different clinical behavior which suggests different intrinsic tumor factors and different p16 expression may be one of these factors [30], [31]. The tumor site was not associated with p16 expression in Smith et al [32] and Ralli et al [27] trials, and in our study (p 0.4, p 0.334, and p 0.840 respectively). Silva et al [33] in their report of a cohort of laryngeal and oropharyngeal HNSCC did not show statistical difference between p16 expression and tumor localization (p16 + in 58.3\% and $52.4 \%$ respectively). The carcinogenesis caused by tobacco and alcohol abuse and HPV infection is known to be synergistic to development of HNSCCs [34-36]. Our study could not find a statistical correlation between smoking tobacco and p16 status similar to findings by Lazarus et al [37] , and Ralli et al [27], but in contrast, Smith et al [32] showed significant association between alcohol and tobacco use $(\mathrm{P}<0.05)$. Tumor grade is a powerful independent predictor of metastases in HNSCC. In our series grade II was the most common in p16 positive (64\%) and p16 negative patients $(58.8 \%)$. In agreement with Yuen et al [31] and Dragomir et al [38] there was no significant correlation between p16 expression and the histologic grade. However, Ralli et al [27] ( $p$ 0.045), Smith et al [32] (p 0.02), and Muirhead et al [39] (p 0.001) showed significant association between 16 status and tumor grade.
P16 protein is an important cell cycle regulator. When underexpressed it causes uncontrolled proliferation of cancer cells, while its overexpression leads to arrest of cell division at G1-S phase thus affects the tumor size and nodal metastases i.e. the stage of tumor [33], [32], [40]. In the current study T3- stage was the most common stage in $\mathrm{p} 16$ $+(16 / 25,64 \%)$ and p $16-(8 / 17,47.1 \%)$ tumors (p 0.756). Similar to our results, Meshman et al [23] could not detect a significant impact of p16 expression on $\mathrm{T}$ - stage of the studied 31 patients with laryngeal and hypophayngeal SCC (p 0.94). Yeun et al [31] showed that weak p16 expression was associated with predominance of $\mathrm{T} 3(55 \%)$ in the studied patients with SCC of the oral cavity, pharynx and larynx (p 0.043).

Lymph nodes are the most common site of spread in HNSCC [41], [42]. In the present study $36 \%$ of positive $p 16$ and $58.8 \%$ of negative p 16 tumors had negative lymph nodes, while $56 \%$ of p $16+$ and $41.1 \%$ of p 16 - tumors had positive nodes ( $p$ 0.454). Our results were in agreement with that of [31], [39] who did not observe a significant correlation. In contrast Ralli et al [27] detected a significant association between lymph nodes involvement in $82.8 \%$ $(53 / 64)$ and p $16+$ staining (p 0.03). Lassen et al [28] studied impact of p 16 status on radiotherapy outcome in OPSCC and non-OPSCC (larynx and hypopharynx) groups of patients. They found a significant correlation between $p$ 16 expression and lymph nodes status in OPSCC patients ( $\mathrm{p}$ $<.0001$ ) but non-significant association in the non-OPSCC group of patients which is similar to our cohort that consisted mostly of laryngeal and hypophayrngeal carcinomas.

The prognostic significance of HPV in subsites outside of the oropharynx is unclear. In the current study p16 expression could not predict a significant impact between the positive $\mathrm{p} 16$ and negative $\mathrm{p} 16$ groups on response to treatment, or DFS and ORS ( $p \geq 0.05)$. Most of the patients received concomitant chemo-radiotherapy $77.2 \%$ (p 2.923), and achieved CR in 59.5\% (p 3.012). The different treatment regimens used in our study in p16 + and p16 - patients had no impact on ORS and DFS ( $p \geq 0.05)$. D'Souza et al [43] studied the prognostic utility of HPV/p16 among nonOPSCC and OPSCC patients across three continents. The authors concluded that positive HVP 16 and/or p16 have no prognostic value on treatment outcome of non-OPSCC. This is consistent with our findings, and several reports suggesting that p16 and /or HPV are not predictors of survival among LSCC [44], [28] and HPSCC [28]. Meshman et al [23] did not support p16 predicting for locoregional control or ORS in the larynx or hypopharynx. Other retrospective studies of p16 in LSCC and HSCC agreed with the negative impact of p16 on outcome [44- 46]. On the contrary, the pooled RTOG analysis of non-OPSCC by Chung et al [16] identified the positive prognostic role of p16 in combined subset analysis. Silver et al [47] evaluated the impact of p16 expression on clinical efficacy of induction low-dose fractionated radiation therapy (LDFRT) with concurrent chemotherapy in patients with locally advanced HNSCC. The authors studied 42 patients with SCC of the larynx, hypopharynx, oral cavity, and oropharynx. They demonstrated that 15/42 (35.7\%) had positive p 16 tumors and their response to induction was 


\section{International Journal of Science and Research (IJSR) \\ ISSN (Online): 2319-7064}

Index Copernicus Value (2015): 78.96 | Impact Factor (2015): 6.391

non-significant (p 0.06). Five-year ORS was $80 \%$ in p16positive patients and $58 \%$ in $\mathrm{p} 16$ negative patients ( $\mathrm{p} 0.025$ ). So the authors concluded that p16 expression affects response to treatment in patients treated with concurrent LDFRT and chemotherapy.

Thus our findings similar to most of literature review failed to prove the hypothesis that the impact of tumor p16-status on treatment regimens, response to treatment and survival outcome also extend to non-OPSCC. This could be partly explained by the small number of studied patients. In agreement with data of Lassen et al [28], our data suggest that p16 positive non-OPSCCs should be considered candidates for enhanced, multimodality treatment protocols similar to p16-negative HNSCCs.

\section{Conclusion}

The prognostic role of $\mathrm{p} 16$ expression has limited utility in non-OPSCCs. We concluded that p16 is not a significant predictor of clinico-pathologic factors and treatment outcome. Interpreting the results of p16 IHC in nonOPSCCs should be done cautiously. It is unclear whether HPV associated non-OPSCCs should be included in the current trials of de-intensification therapy in HPV positive OPSCCs. Consistent with other series Chung et al [16] we recommend further large studies, development of p16 IHC scoring system and improvement of HPV detection methods before broad clinical application of p16 in non-OPSCC.

\section{Conflicts of interest and sources of funding}

None to declare

\section{References}

[1] Venuti A, Badaracco G, Rizzo C, Mafera B, Rahimi S, Vigili M. Presence of HPV in head and neck tumours: high prevalence in tonsillar localization. J Exp Clin Cancer Res. 2004; 23:561-6.

[2] Ragin CC, Taioli E. Survival of squamous cell carcinoma of the head and neck in relation to human papilloma virus infection: review and meta-analysis. Int J Cancer. 2007; 121:1813-20.

[3] O'Rorke MA, Ellison MV, Murray L J, Moran M, James J, Anderson LA. Human Papilloma virus related head and neck cancer survival: a systematic review and meta-analysis. Oral Oncol. 2012; 48:1191-201.

[4] Wilson DD, Shoushtari A, Saylor D et al. P16 is Not a Positive Prognostic Indicator for Hypopharyngeal Squamous Cell Carcinoma Treated with Definitive Chemoradiotherapy. Abstract. I.J. Radiation Onol. Biol. Phys. 2011; 81(2):106.

[5] Evans M, Powell NG. The changing aetiology of head and neck cancer: the role of human papillomavirus. Clin Oncol (R Coll Radiol). 2010; 22: 538-46.

[6] Marur S, D’Souza G, Westra WH, Forastiere AA. HPVassociated head and neck cancer: a virus-related cancer epidemic. Lancet Oncol. 2010; 11: 781-9.

[7] Dayyani F, Etzel CJ, Liu M, et al. Meta-analysis of the impact of human papillomavirus (HPV) on cancer risk and overall survival in head and neck squamous cell carcinomas (HNSCC). Head Neck Oncol. 2010; 2: 15.
[8] Jordan RC, Lingen MW, Perez-Ordonez B, et al. Validation of methods for oropharyngeal cancer HPV status determination in US cooperative group trials. Am J Surg Pathol. 2012; 36:945-54.

[9] Vokes EE, Agrawal N, Seiwert TY. HPV-Associated Head and Neck Cancer. J Natl Cancer Inst. 2015; 107(12): djv344.

[10] Kreimer AR, Clifford GM, Boyle P, Franceschi S. Human papillomavirus types in head and neck squamous cell carcinomas worldwide: a systematic review. Cancer Epidemiol Biomarkers Prev. 2005; 14:467-75.

[11] Isayeva T, Li Y, Maswahu D, Brandwein-Gensler M. Human papillomavirus in non-oropharyngeal head and neck cancers: a systematic literature review. Head Neck Pathol. 2012; 6:S104-20.

[12] Schlecht NF, Brandwein-Gensler M, Nuovo GJ, et al. A comparison of clinically utilized human papillomavirus detection methods in head and neck cancer. Mod Pathol. 2011; 24(10):1295-1305.

[13] Boyer SN, Wazer DE, Band V. E7 protein of human papilloma virus-16 induces degradation of retinoblastoma protein through the ubiquitinproteasome pathway. Cancer Res. 1996; 56(20):46204624.

[14] Liang C, Marsit CJ, McClean MD, et al. Biomarkers of HPV in head and neck squamous cell carcinoma. Cancer Res. 2012; 72 (19):5004-5013.

[15] Lingen MW, Xiao W, Schmitt A, et al. Low etiologic fraction for high-risk human papillomavirus in oral cavity squamous cell carcinomas. Oral Oncol. 2013; 49(1):1-8.

[16]Chung $\mathrm{CH}$, Zhang Q, Kong CS, et al. p16 protein expression and human papillomavirus status as prognostic biomarkers of Non-oropharyngeal head and neck squamous cell carcinoma. J Clin Oncol. 2014; 32:3930-8.

[17] Lewis JS Jr. p16 Immunohistochemistry as a standalone test for risk stratification in oropharyngeal squamous cell carcinoma. Head Neck Pathol. 2012; 6: S75-S82.

[18] Blitzer GC, Smith MA, Harris SL, Kimple R J. Review of the clinical and biologic aspects of human papillomavirus-positive squamous cell carcinomas of the head and neck. Int. J. Radiat. Oncol. Biol. Phys. 2014; 88: 761-770.

[19] Perrone F, Gloghini A, Cortelazzi B, Bossi P, Licitra L, Pilotti S. Isolating p16-positive/HPV-negative oropharyngeal cancer: An effort worth making. Am. J. Surg. Pathol. 2011; 35: 774-777.

[20] Rietbergen MM, Brakenhoff RH, Bloemena E et al. Human papillomavirus detection and comorbidity: Critical issues in selection of patients with oropharyngeal cancer for treatment De-escalation trials. Ann. Oncol. 2013; 24: 2740-2745.

[21] Ang KK, Harris J, Wheeler R, et al. Human papillomavirus and survival of patients with oropharyngeal cancer. N Engl J Med. 2010; 363:24-35.

[22] Lassen P, Eriksen JG, Hamilton-Dutoit S, Tramm T, Alsner J, Overgaard J. Effect of HPV-associated p16INK4A expression on response to radiotherapy and survival in squamous cell carcinoma of the head and neck. J Clin Oncol. 2009; 27: 1992-8. 


\section{International Journal of Science and Research (IJSR) \\ ISSN (Online): 2319-7064 \\ Index Copernicus Value (2015): 78.96 | Impact Factor (2015): 6.391}

[23] Meshman J, Wang PC, Chin R, John MS, Abemayor E, Bhuta S, and Chen AM. Prognostic significance of p16 in squamous cell carcinoma of the larynx and hypopharynx. Am J of Otolaryngology. 2017; 38(1):3137.

[24] Satgunaseelan L, Virk SA, Lum T, Gao K, Clark JR, Gupta R. p16 expression independent of human papillomavirus is associated with lower stage and longer disease-free survival in oral cavity squamous cell carcinoma. Pathology. 2016; 48(5), 441-448.

[25] Chen ZW, Weinreb I, Kamel Reid S, Perez-Ordonez B. Equivocal p16 Immunostaining in Squamous Cell Carcinoma of the Head and Neck: Staining Patterns are Suggestive of HPV Status. Head and Neck Pathol. 2012; 6:422-429.

[26]Deng Z, Hasegawa M, Matayoshi S, et al. Prevalence and clinical features of human papillomavirus in head and neck squamous cell carcinoma in Okinawa, southern Japan. Eur Arch Otorhinolaryngol. 2011; 268(11):1625-1631.

[27] Ralli M, Singh S, Yadav SP, Sharma N, Verma R, Sen R. Assessment and clinicopathological correlation of p16 expression in head and neck squamous cell carcinoma. Journal of Cancer Research and Therapeutics. 2016; 12(1):232-7.

[28] Lassen P, Primdahl H, Johansen J et al On behalf of the Danish Head and Neck Cancer Group (DAHANCA). Impact of HPV-associated p16-expression on radiotherapy outcome in advanced oropharynx and nonoropharynx cancer. Radiotherapy and Oncology. 2014; 113:310-316.

[29] Posner MR, Lorch JH, Goloubeva O, et al. Survival and human papillomavirus in oropharynx cancer in TAX 324: A subset analysis from an international phase III trial. Ann Oncol. 2011; 22:1071-1077.

[30] dos Reis PP, Poli-Frederico RC, dos Santos RM, Nishimoto IN, Kowalski LP, Rogatto SR. Distinct regions of loss of heterozygosity on $22 \mathrm{q}$ in different sites of head and neck squamous cell carcinomas. Med Sci Monit. 2002; 8: BR89- BR94.

[31] Yuen P, Man M, Lam KY, Kwong YL. Clinicopathological significance of p16 gene expression in the surgical treatment of head and neck squamous cell carcinomas. J Clin Pathol. 2002; 55:58-60.

[32] Smith EM, Rubenstein LM, Hoffman H, Haugen TH, Turek LP. Human papillomavirus, p16 and p53 expression associated with survival of head and neck cancer. Infect Agent Cancer. 2010; 5:4.

[33] Silva SD, Nonogaki S, Soares FA, Kowalski LP. p16 (INK4a) has clinicopathological and prognostic impact on oropharynx and larynx squamous cell carcinoma. Brazilian Journal of Medical and Biological Research. 2012; 45: 1327-1333.

[34]Leemans CR, Braakhuis BJ, Brakenhoff RH. The molecular biology of head and neck cancer. Nat. Rev. Cancer. 2011; 11: 9-22.

[35] Ferlay J, Shin HR, Bray F, Forman D, Mathers C, Parkin DM. Estimates of worldwide burden of cancer in 2008: GLOBOCAN 2008. Int. J. Cancer. 2010; 127: 2893-2917.

[36] Kang, H. Kiess A, Chung CH. Emerging biomarkers in head and neck cancer in the era of genomics. Nat. Rev. Clin. Oncol. 2015; 12: 11-26.
[37] Lazarus P, Sheikh SN, Ren Q et al. P53, but not p16 mutations in oral squamous cell carcinomas are associated with specific CYP1A1 and GSTM1 polymorphic genotypes and patient tobacco use. Carcinogenesis. 1998; 19:509-14.

[38] Dragomir LP, Simionescu C, Margaritescu C, Stepan A, Dragomir IM, Popescu MR. P53, p16 and Ki-67 immuno-expression in oral squamous carcinomas. Rom J Morphol Embryol. 2012; 53:89-93.

[39] Muirhead DM, Hoffman HT, Robinson RA. Correlation of clinicopathological features with immunohistochemical expression of cell cycle regulatory proteins p16 and retinoblastoma: Distinct association with keratinization and differentiation in oral cavity squamous cell carcinoma. J Clin Pathol. 2006; 59:711-5.

[40]Ai L, Stephenson KK, Ling W et al. The p16 (CDKN2a/INK4a) tumor suppressor gene in head and neck squamous cell carcinoma: A promoter methylation and protein expression study in 100 cases. Mod Pathol. 2003; 16:944-50

[41] Yuen APW, Lam KY, Chan CLA, et al. Clinicopathological analysis of elective neck dissection for N0 neck of early oral tongue carcinoma. Am J Surg. $1999 ; 177: 90-2$.

[42] Yuen APW, Wei WI, Wong YM, et al. Elective neck dissection versus observation in the surgical treatment of early oral tongue carcinoma. Head Neck 1997; 19:583-8.

[43] D'Soza G, Anantharaman D, Gheit T et al. Effect of HPV on head and neck cancer patient survival, by region and tumor site: A comparison of 1362 cases across three continents. Oral Oncology. 2016; 62: 20 27.

[44] Young RJ, Urban D, Angel C, et al. Frequency and prognostic significance of p16(INK4A) protein overexpression and transcriptionally active human papillomavirus infection in laryngeal squamous cell carcinoma. Br J Cancer. 2015; 112:1098-104.

[45] Wilson DD, Rahimi AS, Saylor DK, et al. P16 not a prognostic marker for hypophayrngeal squamous cell carcinoma. Arch Otolaryngol Head Neck Surg. 2012; 138:556-61.

[46] Salazar CR, Anayannis N, Smith RV, et al. Combined P16 and human papillomavirus testing predicts head and neck cancer survival. Int J Cancer. 2014; 135:2404-12.

[47] Silver NL, Arnold SM, Gleason JF et al. P16INK4a Status and Response to Induction Low-Dose Fractionated Radiation in Advanced Head and Neck Cancer. Annals of Otology, Rhinology \& Laryngology. 2015; 124(9): 714-720. 